\title{
New Results on Soft Spectral in Soft Banach Algebra
}

\author{
Noori F. Al-Mayahi \\ Hayder K. Mohammed \\ Department of Mathematics, College of Computer Science and IT \\ University of Al-Qadisiyah \\ Nfam60@yahoo.com \\ Header.Kadim@gmail.com
}

Recived : 17\4\2018

Revised : //

Accepted : 9\5\2018

Available online : $\quad 5 / 8 / 2018$
DOI: $10.29304 / j q c m .2018 .10 .3 .398$

\begin{abstract}
:
In this paper, concepts of soft character, soft division algebra, soft ideal, soft maximal ideals are introduced .Soft Spectral Radius Formula are introduced and proved, some properties of Soft gelfand algebra are proved.
\end{abstract}

Keywords: soft spectrum, soft spectral radius, soft ideal, soft maximal ideal, soft character, soft regular.

Mathematics Subject Classification: 46S40. 


\section{Introduction}

The soft set theory was first introduced by Russian researcher Molodtsov [1]. Maji, K., Biswas, R. and Roy, R.in [12] introduced many of new concepts of this as an inclusion relation between the soft set, Cagman N. and Enginoglu S. in [3] introduced a new study of a Soft set theory and uniint decision making European J. Oper. Researcher, The application of the soft sets provided a natural framework for generalizing many concepts of topology which is called the soft topological space as initiated by [4] and [11].

Banach Algebra is an important field of functional analysis; therefore Banach algebra structure of soft background was introduced by Thakur R. and Smanta S. in [9]. After that Petroudi S., Sadati S. and Yaghobi A., in [10] obtained a series of new results on Soft banach algebra.

In this paper, concepts of soft character, soft division algebra, soft ideal, soft maximal ideals are introduced .Soft Spectral Radius Formula are introduced and proved, some properties of Soft gelfand algebra are proved.

\section{Preliminaries}

Definition (2.1) [1]:

Let $X$ be a universe set and $E$ be a set of parameters, $P(X)$ the power set of $X$ and

$A \subseteq E$. A pair $(F, A)$ is called soft set over $X$ with respect to $\mathrm{A}$ and $F$ is a mapping given by $F: A \rightarrow$ $P(X)$,

$(F, A)=\{F(e) \in P(X): e \in A\}$.

Definition (2.2) [2]:

(i) A soft set $(F, A)$ over $X$ is called null soft set, denoted by $\widetilde{\emptyset}_{A}$, if for all $e \in A$, we have $F(e)=$ $\emptyset$.

(ii) A soft set $(F, A)$ over $X$ is called absolute soft set and it's denoted by $\tilde{X}_{A}$, if for all

$e \in A$, we have $F(e)=X$.

Definition (2.3) [2,3]:

Let $(F, A)$ and $(G, B)$ be two soft sets over $X$, we say that $(F, A)$ is a soft subset of $(G, B)$ and denoted by $(F, A) \widetilde{\subseteq}(G, B)$, if:

(i) $A \subseteq B$.

(ii) $F(e) \subseteq G(e), \forall e \in A$.

Also, we say that $(F, A)$ and $(G, B)$ are soft equal is denoted by $(F, A)=(G, B)$, if $(F, A) \widetilde{\subseteq}(G, B)$ and $(G, B) \widetilde{\subseteq}(F, A)$.

It is clear that:

(a) $\widetilde{\emptyset}_{A}$ is a soft subset of any soft set $(F, A)$.

(b) Any soft set $(F, A)$ is a soft subset of $\tilde{X}_{A}$.
Definition (2.4) $[2,4]$ :

(i) The intersection of two soft sets $(F, A)$ and $(G, B)$ over a universe $X$ is the soft set $(H, C)=(F, A) \widetilde{\cap}(G, B)$, where $C=A \cap B$ and for all $e \in C$, write $(H, C)=(F, A) \widetilde{\cap}(G, B)$ such that

$H(e)=F(e) \cap G(e)$.

(ii) The union of two soft sets $(F, A)$ and $(G, B)$ over $X$ is the soft set $(G, B)$, where $C=A \cup B$ and $\forall e \in A$ we write

$(H, C)=(F, A) \widetilde{U}(G, B)$ Such that

$H(e)=\left\{\begin{array}{cl}F(e), & \text { if } e \in A \backslash B \\ G(e) & , \text { if } e \in B \backslash A \\ F(e) \cup G(e), & \text { if } e \in A \cap B\end{array}\right.$.

(iii) The difference of two soft sets $(F, A)$

and $(G, A)$ over $X$, denoted by

$(H, C)=(F, A) \widetilde{\backslash}(G, A)$ is defined as

$H(e)=F(e) \backslash G(e)$, for all $e \in A$.

Definition (2.5) [2,5]:

The soft complement of a soft set $(F, A)$ over a universe $X$ is denoted by $(F, A)^{c}$ and it is defined by $(F, A)^{c}=\left(F^{c}, A\right)$, where $F^{c}$ a mapping is given by $F^{c}: A \rightarrow P(X)$,

$F^{c}(e)=X \backslash F(e)$, for all $e \in A$.

i.e. $(F, A)^{c}=\{(e, X \backslash F(e)): \forall e \in A\}$.

It is clear that $\widetilde{\emptyset}_{A}{ }^{c}=\tilde{X}_{A} ; \tilde{X}_{A}{ }^{c}=\widetilde{\emptyset}_{A}$.

Definition (2.6) [6]:

Let $X$ be non-empty set and $E$ be non- empty parameter set. Then a function $\varepsilon: E \rightarrow X$ is said to be a soft element of $X$. A soft element $\varepsilon$ of $X$ is said to belong to soft set $A$ of $X$, which is denoted by $\varepsilon \widetilde{\in} A$, if $\varepsilon(e) \in A(e)$,

$\forall e \in E$. Thus for a soft set $A$ of $X$ with respect to the index set $E$, we have

$A(e)=\{\varepsilon(e): \varepsilon(e) \widetilde{\in} A\}, e \in E\}$.

Definition (2.7) [7]:

Let $\mathbb{C}$ be the set of all complex numbers and $\wp(\mathbb{C})$ be the collection of all non-empty bounded subset of $\mathbb{C}$ and $E$ be a set of parameters.

Then a mapping $F: E \rightarrow \wp(\mathbb{C})$ is called a soft complex set.

It is denoted by $(F, E)$. If in particular $(F, E)$ is a singleton soft set then after identifying $(F, E)$ with the corresponding soft element, it well be called a soft complex number. The soft complex numbers is denoted by $\mathbb{C}(E)$.

Definition (2.8) [7]:

The inverse of any soft complex number $\tilde{r}$, denoted by $\tilde{r}^{-1}$ and defined as $\tilde{r}^{-1}(e)=(\tilde{r}(e))^{-1}$, for all $e \in A$. 
Definition (2.9) [8]:

Let $\tilde{X}$ be the absolute soft vector space i.e., $\tilde{X}(\lambda)=$ $X, \forall \lambda \in E$. Then a mapping

$\|\|:. S E(\tilde{X}) \rightarrow R(E)^{*}$ is said to be soft norm on the soft vector space $\tilde{X}$ if $\|$.$\| satisfies the following$ conditions:

(i) $\left\|\tilde{x}^{e}\right\| \approx \overline{0}$, for all $\tilde{x}^{e} \tilde{\in} \tilde{X}$.

(ii) $\left\|\tilde{x}^{e}\right\|=\overline{0}$ if and only if $\tilde{x}^{e}=\widetilde{\Theta}^{e}$.

(iii) $\left\|\tilde{\lambda} \tilde{x}^{e}\right\| \geq|\tilde{\lambda}|\left\|\tilde{x}^{e}\right\|$, for all $\tilde{x}^{e} \widetilde{\in} \tilde{X}$ and

for every soft scalar $\tilde{\lambda}$.

(iv) $\left\|\tilde{x}^{e}+\tilde{y}^{e}\right\| \widetilde{\leq}\left\|\tilde{x}^{e}\right\|+\left\|\tilde{y}^{e}\right\|$,

for all $\tilde{x}^{e}, \tilde{y}^{e} \widetilde{\in} \tilde{X}$.

The soft vector space $\tilde{X}$ with a soft norm $\|$.$\| on \tilde{X}$ is said to be a soft normed linear space and is denoted by $(\tilde{X},\|\cdot\|)$.

Definition (2.10) [9]:

A sequence of soft elements $\left\{\tilde{x}_{n}^{e}\right\}$ in a soft normed linear space $(\tilde{X},\|\cdot\|)$. is said to be convergent and converges to a soft element $\tilde{x}^{e}$ if $\left\|\tilde{x}_{n}^{e}-\tilde{x}^{e}\right\| \rightarrow \overline{0}$ as $n \rightarrow \infty$ This means for every $\tilde{\epsilon} \geq \overline{0}$, chosen arbitrary, there exists a natural number $=N(\epsilon)$, such that $\overline{0} \widetilde{\leq}\left\|\tilde{x}_{n}^{e}-\tilde{x}^{e}\right\| \widetilde{\leq} \tilde{\epsilon}$, whenever $n>N$. We denote this by $\tilde{x}_{n}^{e} \rightarrow \tilde{x}^{e}$ as $n \rightarrow \infty$ or $\lim _{n \rightarrow \infty} \tilde{x}_{n}^{e}=\tilde{x}^{e} . \tilde{x}^{e}$ is said to be the limit of the sequence $\tilde{x}^{e}{ }_{n}$ as $n \rightarrow \infty$.

Definition (2.11) [9]:

Let $T: S E(\tilde{X}) \rightarrow S E(\tilde{Y})$ be an operator. Then $T$ is said to be soft linear if

$T\left(\tilde{\lambda}_{1} \tilde{x}^{e}{ }_{1}+\tilde{\lambda}_{2} \tilde{x}_{2}{ }_{2}\right)=\tilde{\lambda}_{1} T\left(\tilde{x}^{e}{ }_{1}\right)+\tilde{\lambda}_{2} T\left(\tilde{x}^{e}{ }_{2}\right)$

for every soft scalar $\tilde{\lambda}$ and $\tilde{x}^{e}{ }_{1}, \tilde{x}^{e}{ }_{2} \widetilde{\in} \tilde{X}$.

Definition (2.12) [11]:

Let $(F, E),(G, E)$ are soft sets in $S(X)_{E}$, a soft mapping

$\mathrm{f}:(\mathrm{F}, \mathrm{E}) \stackrel{\rightarrow}{\rightarrow}(\mathrm{G}, \mathrm{E})$ is said to be (soft injective )if each soft element in $(F, E)$ is related to a different in $(\mathrm{G}, \mathrm{E})$.

More formally $\mathrm{f}\left(\tilde{\mathrm{x}}_{1}\right)=\mathrm{f}\left(\widetilde{\mathrm{x}}_{2}\right)$ implies $\tilde{\mathrm{x}}_{1}=\tilde{\mathrm{x}}_{2}$.

Definition (2.13) [11]:

Let $(F, E),(G, E)$ are soft sets in $S(X)_{E}$, a soft mapping

$\mathrm{f}:(\mathrm{F}, \mathrm{E}) \stackrel{\sim}{\rightarrow}(\mathrm{G}, \mathrm{E})$ is said to be (soft surjective) if for every soft element $\tilde{y}$ in $(\mathrm{G}, \mathrm{E})$, there is a soft element $\tilde{\mathrm{x}}$ in $(F, E)$ such that $\mathrm{f}(\tilde{\mathrm{x}})=\tilde{\mathrm{y}}$,

[i.e. when the soft range equals to the soft image $\mathrm{f}((\mathrm{F}, \mathrm{E}))=(\mathrm{G}, \mathrm{E})]$.

Definition (2.14 [9]:

Let $V$ be algebra over a scalar field $\mathbb{R}$ and let $E$ be the parameter set and $F_{E}$ be a soft set over $V$. Now, $F_{E}$ is called soft algebra (in short $S A$ ) of $V$ over $K(E)$ if $F(e)$ is a sub algebra of $V$ for all $e \in E$. It is very easy to see that in $S A$. the soft elements satisfy the properties:

(i) $\left(\tilde{x}^{e} \tilde{y}^{e}\right) \tilde{z}^{e}=\tilde{x}^{e}\left(\tilde{y}^{e} \tilde{z}^{e}\right)$.

(ii) $\tilde{x}^{e}\left(\tilde{y}^{e} \widetilde{+} \tilde{z}^{e}\right)=\tilde{x}^{e} \tilde{y}^{e} \widetilde{+} \tilde{x}^{e} \tilde{z}^{e} ;\left(\tilde{y}^{e} \widetilde{+} \tilde{z}^{e}\right) \tilde{x}^{e}=$ $\tilde{y}^{e} \tilde{x}^{e} \tilde{+} \tilde{z}^{e} \tilde{x}^{e}$. (iii) $\tilde{\alpha}\left(\tilde{x}^{e} \tilde{y}^{e}\right)=\left(\tilde{\alpha} \tilde{x}^{e}\right) \tilde{y}^{e}=\tilde{x}^{e}\left(\tilde{\alpha} \tilde{y}^{e}\right)$, where for all $\tilde{x}^{e}, \tilde{y}^{e}, \tilde{z}^{e} \widetilde{\in} F_{E}$ and for any soft scalar $\tilde{\alpha}$. If $F_{E}$ is also soft Banach space w.r.t. a soft norm that satisfies the inequality $\left\|\tilde{x}^{e} \tilde{y}^{e}\right\| \leq\left\|\tilde{x}^{e}\right\|\left\|\tilde{y}^{e}\right\|$ and $F_{E}$ is contains the unitary element $\tilde{u}^{e}$ such that $\tilde{x}^{e} \tilde{u}^{e}=\tilde{u}^{e} \tilde{x}^{e}=\tilde{x}^{e}$ with $\left\|\tilde{u}^{e}\right\|=\overline{1}$, then is called soft Banach algebra (in short $S B A$ ).

Definition (2.15) [10]:

Let $\mathfrak{A}$ be a $(S B A)$ with $\tilde{u}^{e}$ and $\tilde{\mathrm{x}}^{\mathrm{e}} \widetilde{\in} \mathfrak{U}$. Then the soft set $\delta\left(\widetilde{\mathrm{x}}^{\mathrm{e}}\right)=\left\{\tilde{\lambda} \widetilde{\in} \mathbb{R}(\mathrm{E}):\left(\tilde{\mathrm{x}}^{\mathrm{e}} \simeq \tilde{\lambda} \widetilde{\mathrm{u}}^{\mathrm{e}}\right)\right.$

is soft singular $\}$ is called soft spectrum of $\tilde{x}^{e} \widetilde{\in} \mathfrak{A}$.

Definition (2.16): [10]

We denote the soft spectral radius of $\tilde{\mathrm{x}}^{\mathrm{e}}$ by $\mathrm{r}\left(\tilde{\mathrm{x}}^{\mathrm{e}}\right)$ and define it by

$\mathrm{r}\left(\widetilde{\mathrm{x}}^{\mathrm{e}}\right)=\operatorname{Sup}\left\{|\widetilde{\alpha}|: \widetilde{\alpha} \widetilde{\in} \delta\left(\widetilde{\mathrm{x}}^{\mathrm{e}}\right)\right\}$.

\section{Results}

Theorem (3.1):

Let $\mathfrak{A}$ be (SBA) with identity element $(\overline{1})$ and $\widetilde{\mathrm{X}}^{\mathrm{e}} \widetilde{\in} \mathfrak{A}$, then $\delta\left(\widetilde{\mathrm{x}}^{\mathrm{e}}\right)=\left(\delta\left(\widetilde{\mathrm{x}}^{\mathrm{e}}\right)\right)^{\mathrm{n}}$.

Proof:

Let $\widetilde{\alpha} \widetilde{\in} \delta\left(\widetilde{\mathrm{x}}^{\mathrm{e}}\right)$, then $\left(\widetilde{\mathrm{x}}^{\mathrm{e}}-\widetilde{\alpha} . \overline{1}\right) \widetilde{\epsilon} \mathrm{S}_{\mathrm{E}}$

Consider

$$
\tilde{\mathrm{x}}^{\mathrm{e}^{\mathrm{n}}}-\widetilde{\alpha} .=\left(\tilde{\mathrm{x}}^{\mathrm{e}}-\widetilde{\alpha}_{1} \cdot \overline{1}\right), \ldots \ldots,\left(\widetilde{\mathrm{x}}^{\mathrm{e}}-\widetilde{\alpha}_{\mathrm{n}} \cdot \overline{1}\right)
$$

Where $\widetilde{\alpha}_{1}, \widetilde{\alpha}_{2}, \ldots \ldots, \widetilde{\alpha}_{\mathrm{n}}$ are roots of $\tilde{\mathrm{x}}^{\mathrm{e}}-\widetilde{\alpha} . \overline{1}$

Let $\widetilde{\alpha} \widetilde{\in} \delta\left(\widetilde{\mathrm{x}}^{\mathrm{e}}\right)$, i.e. $\widetilde{\mathrm{x}}^{\mathrm{e}}-\widetilde{\alpha} \cdot \overline{1} \widetilde{\in} \mathrm{S}_{\mathrm{E}}$

then there is at least one i such that $\left(\tilde{\mathrm{x}}^{\mathrm{e}}-\widetilde{\alpha}_{\mathrm{i}} \cdot \overline{1}\right) \widetilde{\in} \mathrm{S}_{\mathrm{E}}$, hence $\widetilde{\alpha}_{\mathrm{i}} \widetilde{\in} \delta\left(\widetilde{\mathrm{x}}^{\mathrm{e}}\right)$ So $\widetilde{\alpha}_{\mathrm{i}}{ }^{\mathrm{n}} \widetilde{\in}\left(\delta\left(\widetilde{\mathrm{x}}^{\mathrm{e}}\right)\right)^{\mathrm{n}}$, then $\widetilde{\alpha} \widetilde{\epsilon}\left(\delta\left(\widetilde{\mathrm{x}}^{\mathrm{e}}\right)\right)^{\mathrm{n}}$

Hence $\delta\left(\widetilde{\mathrm{x}}^{\mathrm{e}}\right) \widetilde{\simeq}\left(\delta\left(\widetilde{\mathrm{x}}^{\mathrm{e}}\right)\right)^{\mathrm{n}}$

Let $\widetilde{\alpha}^{\mathrm{n}} \widetilde{\in}\left(\delta\left(\widetilde{\mathrm{x}}^{\mathrm{e}}\right)\right)^{\mathrm{n}}$, where $\widetilde{\alpha} \widetilde{\in} \delta\left(\tilde{\mathrm{x}}^{\mathrm{e}}\right)$.

Note that $\left(\tilde{\mathrm{x}}^{\mathrm{e}}-\widetilde{\alpha} . \overline{1}\right)$ is soft factor of $\tilde{\mathrm{x}}^{\mathrm{e}^{\mathrm{n}}}-\widetilde{\alpha}^{\mathrm{n}} \cdot \overline{1}, \operatorname{hence}\left(\tilde{\mathrm{x}}^{\mathrm{e}}-\widetilde{\alpha} \cdot \overline{1}\right) \widetilde{\epsilon} \mathrm{S}_{\mathrm{E}}$ $\widetilde{\mathrm{x}}^{\mathrm{e}}-\widetilde{\alpha}^{\mathrm{n}} \cdot \overline{1} \widetilde{\epsilon} \mathrm{S}_{\mathrm{E}}$ So $\widetilde{\alpha}^{\mathrm{n}} \widetilde{\in} \delta\left(\widetilde{\mathrm{x}}^{\mathrm{e}}\right)$.

Then $\left(\delta\left(\tilde{\mathrm{x}}^{\mathrm{e}}\right)\right)^{\mathrm{n}} \widetilde{\subseteq} \delta\left(\widetilde{\mathrm{x}}^{\mathrm{e}}{ }^{\mathrm{n}}\right) \ldots \ldots \ldots(2)$

by (1) and (2), we have $\delta\left(\widetilde{\mathrm{x}}^{\mathrm{e}}\right)=\left(\delta\left(\widetilde{\mathrm{x}}^{\mathrm{e}}\right)\right)^{\mathrm{n}}$.

Theorem (3.2):

Let $\mathfrak{A}$ be (SBA) with identity element $(\overline{1})$ and $\widetilde{\mathrm{X}}^{\mathrm{e}} \widetilde{\in} \mathfrak{U}$, then $r\left(\widetilde{\mathrm{x}}^{\mathrm{e}}\right)=\left(\mathrm{r}\left(\widetilde{\mathrm{x}}^{\mathrm{e}}\right)\right)^{\mathrm{n}}$.

Proof:

Since $r\left(\widetilde{\mathrm{x}}^{\mathrm{e}}\right)=\operatorname{Sup}\left\{|\widetilde{\alpha}|: \widetilde{\alpha} \widetilde{\in} \delta\left(\widetilde{\mathrm{x}}^{\mathrm{e}}\right)\right\}$.

Then $\left(\mathrm{r}\left(\widetilde{\mathrm{x}}^{\mathrm{e}}\right)\right)^{\mathrm{n}}=\left(\operatorname{Sup}\left\{|\widetilde{\alpha}|: \widetilde{\alpha} \widetilde{\in} \delta\left(\tilde{\mathrm{x}}^{\mathrm{e}}\right)\right\}\right)^{\mathrm{n}}$.

Hence $\left(\mathrm{r}\left(\tilde{\mathrm{x}}^{\mathrm{e}}\right)\right)^{\mathrm{n}}=\operatorname{Sup}\left\{|\widetilde{\alpha}|^{\mathrm{n}}: \widetilde{\alpha} \widetilde{\in} \delta\left(\widetilde{\mathrm{x}}^{\mathrm{e}}\right)\right\}$.

Now

$\mathrm{r}\left(\widetilde{\mathrm{x}}^{\mathrm{e}^{\mathrm{n}}}\right)=\operatorname{Sup}\left\{|\widetilde{\alpha}|: \widetilde{\alpha} \widetilde{\in} \delta\left(\tilde{\mathrm{x}}^{\mathrm{en}}\right)\right\}$

$=\operatorname{Sup}\left\{|\widetilde{\alpha}|: \widetilde{\alpha} \widetilde{\in}\left(\delta\left(\tilde{\mathrm{x}}^{\mathrm{e}}\right)\right)^{\mathrm{n}}\right\}$

$=\operatorname{Sup}\left\{\left|\widetilde{\beta}^{\mathrm{n}}\right|: \tilde{\beta} \widetilde{\in} \delta\left(\tilde{\mathrm{x}}^{\mathrm{e}}\right)\right\}$, for some

$\widetilde{\beta} \widetilde{\in} \delta\left(\widetilde{\mathrm{x}}^{\mathrm{e}}\right)$

$=\left(\operatorname{Sup}\left\{|\tilde{\beta}|: \tilde{\beta} \widetilde{\in} \delta\left(\tilde{\mathrm{x}}^{\mathrm{e}}\right)\right\}\right)^{\mathrm{n}}$

$=\left(\mathrm{r}\left(\widetilde{\mathrm{x}}^{\mathrm{e}}\right)\right)^{\mathrm{n}}$, as $\left|\widetilde{\beta}^{\mathrm{n}}\right|=|\widetilde{\beta}|^{\mathrm{n}}, \widetilde{\beta} \widetilde{\in} \mathbb{C}(\mathrm{E})$.

Theorem (3.3) [10]:

Let $\mathfrak{A}$ be a $(S B A)$ with $\tilde{u}^{e}$.

Then every $\tilde{x}^{e} \widetilde{\in} \mathfrak{U}$ for $\left\|\tilde{u}^{e} \simeq \tilde{x}^{e}\right\| \widetilde{\leq} \overline{1}$ is soft regular and $\tilde{x}^{e^{-1}}=\sum_{n=0}^{\infty}\left(\tilde{u}^{e} \simeq \tilde{x}^{e}\right)^{n}$. 
Theorem (3.4): (Soft Spectral Radius Formula)

Let $\mathfrak{A}$ be (SBA) with soft identity element and $\tilde{\mathrm{X}}^{\mathrm{e}} \widetilde{\in} \mathfrak{A}$, then $\lim _{\mathrm{n} \rightarrow \infty}\left\|\tilde{\mathrm{X}}^{\mathrm{e}}\right\|^{\frac{1}{\mathrm{n}}}$ exists and $\mathrm{r}\left(\tilde{\mathrm{X}}^{\mathrm{e}}\right)=$ $\lim _{n \rightarrow \infty}\left\|\tilde{x}^{e^{n}}\right\|^{\frac{1}{n}}$.

\section{Proof:}

Let $g\left(\tilde{\mathrm{x}}^{\mathrm{e}}\right)=\lim _{\mathrm{n} \rightarrow \infty}\left\|\tilde{\mathrm{x}}^{\mathrm{e} \mathrm{n}}\right\|^{\frac{1}{\mathrm{n}}}$.For

$\epsilon>0$ then there is $\mathrm{k}$ Such that

$\left\|\tilde{\mathrm{x}}^{\mathrm{e}}\right\|^{\frac{1}{\mathrm{k}}} \approx \mathrm{\gtrless}\left(\tilde{\mathrm{x}}^{\mathrm{e}}\right) \widetilde{+} \epsilon$.

for any $\mathrm{n} \in \mathbb{N}$ we have

$\mathrm{n}=\alpha \mathrm{k}+\beta, 0 \leq \beta<k, \mathrm{k}<\mathrm{n}, \alpha, \beta \in \mathbb{Z}^{+}$

$\overline{1}=\alpha \frac{\mathrm{k}}{\mathrm{n}}+\frac{\beta}{\mathrm{n}}$, as $\mathrm{k}<\mathrm{n} \Rightarrow \frac{\mathrm{k}}{\mathrm{n}} \rightarrow 0, \frac{\beta}{\mathrm{n}} \rightarrow 0$ as $\mathrm{n} \rightarrow \infty \Rightarrow \mathrm{k} \frac{\alpha}{\mathrm{n}} \rightarrow \overline{1} \Rightarrow \frac{\alpha}{\mathrm{n}} \rightarrow \frac{\overline{1}}{\mathrm{k}}$.

Now

$\left\|\tilde{\mathrm{x}}^{\mathrm{e}}\right\|^{\frac{1}{\mathrm{n}}}=\left\|\left(\tilde{\mathrm{x}}^{\mathrm{e}^{\mathrm{k}}}\right)^{\alpha \sim} \cdot \tilde{\mathrm{x}}^{\mathrm{e} \beta}\right\|_{1}^{\frac{1}{\mathrm{n}}}$

$\widetilde{\leq}\left\|\left(\tilde{\mathrm{x}}^{\mathrm{e}^{\mathrm{k}}}\right)^{\alpha}\right\|^{\frac{1}{\mathrm{n}}} \sim\left\|\tilde{\mathrm{x}}^{\mathrm{e} \beta}\right\|^{\frac{1}{\mathrm{n}}} \widetilde{\leq}\left\|\tilde{\mathrm{x}}^{\mathrm{e}^{\mathrm{k}}}\right\|^{\frac{\alpha}{\mathrm{n}}} \sim\left\|\tilde{\mathrm{x}}^{\mathrm{e}}\right\|^{\frac{\beta}{\mathrm{n}}}$

Since $\left\|\tilde{\mathrm{x}}^{\mathrm{e}^{\mathrm{k}}}\right\|^{\frac{\alpha}{\mathrm{n}}} \rightarrow\left\|\tilde{\mathrm{x}}^{\mathrm{e}^{\mathrm{k}}}\right\|^{\frac{1}{\mathrm{k}}}$ and $\left\|\tilde{\mathrm{x}}^{\mathrm{e}}\right\|^{\frac{\beta}{\mathrm{n}}} \rightarrow \overline{1}$

$\Rightarrow\left\|\tilde{\mathrm{x}}^{\mathrm{e} \mathrm{n}}\right\|^{\frac{1}{\mathrm{n}}} \widetilde{\leq}\left\|\tilde{\mathrm{x}}^{\mathrm{e}^{\mathrm{k}}}\right\|^{\frac{1}{\mathrm{k}}}$

$\Rightarrow \lim _{\mathrm{n} \rightarrow \infty}\left\|\tilde{\mathrm{x}}^{\mathrm{e}^{\mathrm{n}}}\right\|^{\frac{1}{\mathrm{n}}} \widetilde{\leq}\left\|\tilde{\mathrm{x}}^{\mathrm{e}^{\mathrm{k}}}\right\|^{\frac{1}{\mathrm{k}}}$

Then from $(*)$, we have

$\left\|\tilde{\mathrm{x}}^{\mathrm{e}}\right\|^{\frac{1}{\mathrm{n}}} \widetilde{<} g\left(\tilde{\mathrm{x}}^{\mathrm{e}}\right) \widetilde{+} \epsilon, \forall \mathrm{n}$

Note that $g\left(\widetilde{\mathrm{x}}^{\mathrm{e}}\right) \widetilde{\leq}\left\|\tilde{\mathrm{x}}^{\mathrm{e}}\right\|^{\frac{1}{\mathrm{n}}}, \forall \mathrm{n}$

$\Rightarrow g\left(\tilde{\mathrm{x}}^{\mathrm{e}}\right) \widetilde{\leq}\left\|\tilde{\mathrm{x}}^{\mathrm{e}}\right\|^{\frac{1}{\mathrm{n}}} \widetilde{<} g\left(\tilde{\mathrm{x}}^{\mathrm{e}}\right) \widetilde{+} \epsilon$

$\Rightarrow \lim _{\mathrm{n} \rightarrow \infty}\left\|\tilde{\mathrm{x}}^{\mathrm{e}}\right\|^{\frac{1}{\mathrm{n}}}=g\left(\tilde{\mathrm{x}}^{\mathrm{e}}\right)$

Hence the limit exists.

Now to show that $r\left(\tilde{\mathrm{x}}^{\mathrm{e}}\right)=\lim _{\mathrm{n} \rightarrow \infty}\left\|\tilde{\mathrm{x}}^{\mathrm{e} \mathrm{n}}\right\|^{\frac{1}{\mathrm{n}}}$, since $\mathrm{r}\left(\widetilde{\mathrm{x}}^{\mathrm{e}}\right)=\operatorname{Sup}\left\{|\widetilde{\alpha}|: \widetilde{\alpha} \widetilde{\in} \delta\left(\widetilde{\mathrm{x}}^{\mathrm{e}}\right)\right\}$ and $|\widetilde{\alpha}| \widetilde{\leq}\left\|\widetilde{\mathrm{x}}^{\mathrm{e}}\right\|$, for $\widetilde{\alpha} \widetilde{\in} \delta\left(\widetilde{\mathrm{x}}^{\mathrm{e}}\right) \Rightarrow\left|\widetilde{\alpha}^{\mathrm{n}}\right| \widetilde{\leq}\left\|\widetilde{\mathrm{x}}^{\mathrm{e}}\right\|$

$\Rightarrow|\widetilde{\alpha}|^{\mathrm{n}} \widetilde{\leq}\left\|\tilde{\mathrm{x}}^{\mathrm{e}}\right\|$ by $\left(\delta\left(\widetilde{\mathrm{x}}^{\mathrm{e}}\right)\right)=\left(\delta\left(\tilde{\mathrm{x}}^{\mathrm{e}}\right)\right)^{\mathrm{n}}$

$\Rightarrow|\widetilde{\alpha}| \widetilde{\leq}\left\|\widetilde{\mathrm{x}}^{\mathrm{e}}\right\|^{\frac{1}{\mathrm{n}}}, \forall \mathrm{n}$

$\therefore \operatorname{Sup}\left\{|\widetilde{\alpha}|: \widetilde{\alpha} \widetilde{\in} \delta\left(\widetilde{\mathrm{x}}^{\mathrm{e}}\right)\right\} \widetilde{\leq}\left\|\tilde{\mathrm{x}}^{\mathrm{e}^{\mathrm{n}}}\right\|^{\frac{1}{\mathrm{n}}}, \forall \mathrm{n}$

$\Rightarrow \mathrm{r}\left(\tilde{\mathrm{x}}^{\mathrm{e}}\right) \underset{\leq}{\widetilde{s}} \mathrm{~g}\left(\tilde{\mathrm{x}}^{\mathrm{e}}\right)$.

Let $\widetilde{\alpha} \widetilde{\in} \mathbb{C}(\mathrm{E})$ with $|\widetilde{\alpha}| \widetilde{\geq}\left\|\widetilde{\mathrm{x}}^{\mathrm{e}}\right\|$.

i.e $\quad \widetilde{\alpha} \widetilde{\notin} \quad \delta\left(\tilde{\mathrm{x}}^{\mathrm{e}}\right) \quad \Rightarrow \quad\left\|\frac{\tilde{\mathrm{x}}^{\mathrm{e}}}{\widetilde{\alpha}}\right\| \widetilde{\leq} \overline{1}$ $\Rightarrow\left\|\tilde{u}^{e} \simeq\left(\tilde{u}^{e} \simeq \frac{\tilde{x}^{\mathrm{e}}}{\widetilde{\alpha}}\right)\right\| \widetilde{\leq}$ and by theorem (3.3), we get $\left(\tilde{u}^{e} \simeq \frac{\tilde{\mathrm{x}}^{\mathrm{e}}}{\widetilde{\alpha}}\right)$ is soft regular and $\left(\tilde{u}^{e} \simeq \frac{\tilde{\mathrm{x}}^{\mathrm{e}}}{\widetilde{\alpha}}\right)^{-1}=$ $\sum_{\mathrm{n}=0}^{\infty}\left(\frac{\tilde{\mathrm{x}}^{\mathrm{e}}}{\widetilde{\alpha}}\right)^{\mathrm{n}}$

$\Rightarrow\left(\frac{\widetilde{\alpha} \widetilde{\tilde{x}^{\mathrm{e}}}}{\widetilde{\alpha}}\right)^{-1}=\sum_{\mathrm{n}=0}^{\infty}\left(\frac{\tilde{\mathrm{x}}^{\mathrm{e}}}{\widetilde{\alpha}}\right)^{\mathrm{n}}$

$\Rightarrow\left(\tilde{\mathrm{x}}^{\mathrm{e}} \simeq \widetilde{\alpha}\right)^{-1}=-\sum_{\mathrm{n}=0}^{\infty} \frac{\tilde{\mathrm{x}}^{\mathrm{e}^{\mathrm{n}}}}{\widetilde{\alpha}^{\mathrm{n}+1}}$

$=\widetilde{\mathrm{x}}^{\mathrm{e}}(\widetilde{\alpha})$. This series $(*)$ is convergent because it is absolutely convergent.

Now, let $\mathbb{f} \widetilde{\in} \mathrm{A}^{*}=$ set of all soft bounded linear functional
$\Rightarrow \mathbb{f}\left(\widetilde{\mathrm{X}}^{\mathrm{e}}(\widetilde{\alpha})\right)=\sum_{\mathrm{n}=0}^{\infty} \frac{\mathbb{f}\left(\tilde{\mathrm{x}}^{\mathrm{n}}\right)}{\widetilde{\alpha}^{\mathrm{n}+1}}$ and again $\sum_{\mathrm{n}=0}^{\infty} \frac{\mathbb{f}\left(\tilde{\mathrm{x}}^{\mathrm{n}}\right)}{\widetilde{\alpha}^{\mathrm{n}+1}}$ is also soft convergent for $|\widetilde{\alpha}| \geq\left\|\widetilde{\mathrm{X}}^{\mathrm{e}}\right\| \geq \mathrm{r}\left(\widetilde{\mathrm{x}}^{\mathrm{e}}\right) \Rightarrow$ $\sum_{\mathrm{n}=0}^{\infty} \frac{\left|\mathbb{f}\left(\tilde{\mathrm{x}}^{\mathrm{n}}\right)\right|}{\widetilde{\alpha}^{\mathrm{n}+1}} \widetilde{\leq} \sum_{\mathrm{n}=0}^{\infty} \frac{\left|\mathbb{f}\left(\tilde{\mathrm{x}}^{\mathrm{e}}\right)\right|^{\mathrm{n}}}{\widetilde{\alpha}^{\mathrm{n}+1}}$ and since soft bounded

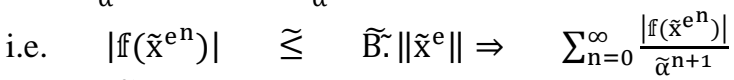
$\widetilde{\leq} \sum_{\mathrm{n}=0}^{\infty} \frac{\widetilde{\mathrm{B}}^{\mathrm{n}} \sim\left\|\widetilde{\mathrm{x}}^{\mathrm{e}}\right\|^{\mathrm{n}}}{\widetilde{\alpha}^{\mathrm{n}+1}}$

We know that for all soft bounded linear functional $\Rightarrow \mathbb{f}\left(\widetilde{\mathrm{X}}^{\mathrm{e}}(\widetilde{\alpha})\right)$ is analytic for

$|\widetilde{\alpha}| \geq \mathrm{r}\left(\tilde{\mathrm{x}}^{\mathrm{e}}\right)$

$\therefore$ It has a series representation

$\Rightarrow \mathbb{f}\left(\widetilde{\mathrm{x}}^{\mathrm{e}}(\widetilde{\alpha})\right)=\sum_{\mathrm{n}=0}^{\infty} \frac{\mathbb{f}\left(\tilde{\mathrm{x}}^{\mathrm{e}}\right)}{\widetilde{\alpha}^{\mathrm{n}+1}}$ exists

$\Rightarrow \frac{\mathbb{f}\left(\widetilde{\mathrm{x}}^{\mathrm{e}}\right)}{\widetilde{\alpha}^{\mathrm{n}+1}} \rightarrow \theta$ as $\mathrm{n} \rightarrow \infty \quad$ i.e $\quad\left\{\frac{\mathbb{f}\left(\widetilde{\mathrm{x}}^{\mathrm{e}}\right)}{\widetilde{\alpha}^{\mathrm{n}+1}}\right\} \rightarrow \theta \quad$ as $\mathrm{n} \rightarrow \infty$.

the soft set $\widetilde{E}=\left\{\frac{\widetilde{x}^{e^{n}}}{\widetilde{\alpha}^{n+1}}\right\}$ is soft bounded $\Rightarrow$ i.e

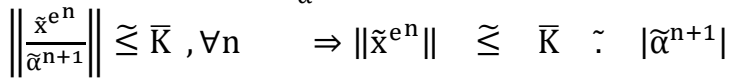
$=\overline{\mathrm{K}} \sim|\widetilde{\alpha}|^{\sim}|\widetilde{\alpha}|^{\mathrm{n}} \Rightarrow\left\|\tilde{\mathrm{x}}^{\mathrm{e}^{\mathrm{n}}}\right\|^{\frac{1}{\mathrm{n}}} \widetilde{\leq}(\overline{\mathrm{K}} \tilde{.} \mid \widetilde{\alpha})^{\frac{1}{\mathrm{n}}} \quad \sim|\widetilde{\alpha}|$ $\Rightarrow \lim _{\mathrm{n} \rightarrow \infty}\left\|\tilde{\mathrm{X}}^{\mathrm{e}^{\mathrm{n}}}\right\|^{\frac{1}{\mathrm{n}}} \widetilde{\leq} \lim _{\mathrm{n} \rightarrow \infty}(\overline{\mathrm{K}} \tilde{\sim}|\widetilde{\alpha}|)^{\frac{1}{\mathrm{n}}} \tilde{\cdot}|\widetilde{\alpha}| \quad \Rightarrow$ $\lim _{\mathrm{n} \rightarrow \infty}\left\|\tilde{\mathrm{x}}^{\mathrm{e}}\right\|^{\frac{1}{\mathrm{n}}} \widetilde{\leq}|\widetilde{\alpha}|$ where $|\widetilde{\alpha}| \geq \mathrm{r}\left(\tilde{\mathrm{x}}^{\mathrm{e}}\right)$, hence $\mathrm{r}\left(\tilde{\mathrm{x}}^{\mathrm{e}}\right) \geq g\left(\widetilde{\mathrm{x}}^{\mathrm{e}}\right)$

From (1) and (2)

We get $r\left(\tilde{\mathrm{x}}^{\mathrm{e}}\right)=\lim _{\mathrm{n} \rightarrow \infty}\left\|\tilde{\mathrm{x}}^{\mathrm{e}^{\mathrm{n}}}\right\|^{\frac{1}{n}}$.

Definition (3.5):

Let $\mathfrak{A}$ be (S. B. A) over $\mathbb{K}(\mathrm{E})$. A soft character (in short S.char) of $\mathfrak{A}$ is a nontrivial

Soft algebra soft homomorphism

$\widetilde{\Im}: \mathfrak{U} \rightarrow \mathbb{K}(\mathrm{E})$, which means that:

(i) $\widetilde{\mathfrak{I}}$ is soft linear.

(ii) $\widetilde{\Im}$ is soft multiplicative.

Remark (3.6):

If $\mathfrak{A}$ has $\tilde{u}^{\mathrm{e}}$, then the fact that $\widetilde{\mathfrak{I}}$ is a nontrivial force the equality $\widetilde{\mathfrak{I}}\left(\tilde{\mathrm{u}}^{\mathrm{e}}\right)=\overline{1}$. we then define S. $\operatorname{char}(\mathfrak{U})=\{\widetilde{\mathfrak{I}}: \mathfrak{A} \rightarrow \mathbb{K}(\mathrm{E}): \widetilde{\mathfrak{I}}$ soft character $\}$.

Definition (3.7) [9]: Let $\mathfrak{U}$ is a $(S B A)$ with $\tilde{u}^{e}$. Then $\tilde{x}^{e} \widetilde{\in} \mathfrak{A}$ is said to be soft regular, if $\tilde{x}^{e}$ is invertible (i.e. there a soft element $\tilde{x}^{e^{-1}}$ called the inverse of $\tilde{x}^{e}$, such that $\tilde{x}^{e}\left(\tilde{x}^{e}\right)^{-1}=\left(\tilde{x}^{e}\right)^{-1} \tilde{x}^{e}=$ $\left.\tilde{u}^{e}\right)$.

\section{Definition (3.8)}

A soft algebra $\mathfrak{A}$ is said to be soft division algebra if $\tilde{x}^{e} \widetilde{\in} \mathfrak{A}$ and $\tilde{x}^{e} \neq \Theta^{e}$ is soft regular.

Theorem (3.9) (Soft gelfand algebra):

Every SBA and commutative with $\tilde{\mathrm{u}}^{\mathrm{e}}$ over $\mathbb{C}(\mathrm{E})$ which is soft division algebra is soft isomorphic with $\mathbb{C}(\mathrm{E})$. 


\section{Definition (3.10):}

(i) A soft set I(E) of soft elements of $\mathfrak{A}$ (S.BA) is called soft left ideal if it is no null soft set and has the following properties:

(a)If $\tilde{\mathrm{x}}^{\mathrm{e}}, \tilde{\mathrm{y}}^{\mathrm{e}} \widetilde{\in} \mathrm{I}(\mathrm{E})$ then $\tilde{\mathrm{x}}^{\mathrm{e}} \widetilde{千} \tilde{\mathrm{y}}^{\mathrm{e}} \widetilde{\in} \mathrm{I}(\mathrm{E})$;

(b)If $\widetilde{\mathrm{x}}^{\mathrm{e}} \widetilde{\in} \mathrm{I}(\mathrm{E})$ and $\widetilde{\alpha} \widetilde{\in} \mathbb{C}(\mathrm{E})$ then $\widetilde{\alpha} \widetilde{\mathrm{x}}^{\mathrm{e}} \widetilde{\in} \mathrm{I}(\mathrm{E})$;

(c) If $\tilde{\mathrm{X}}^{\mathrm{e}} \widetilde{\in} \mathrm{I}(\mathrm{E})$ and $\tilde{\mathrm{r}}^{\mathrm{e}} \widetilde{\in} \mathfrak{A}$ then $\tilde{\mathrm{r}}^{\mathrm{e}} \sim \widetilde{\mathrm{x}}^{\mathrm{e}} \widetilde{\in} \mathrm{I}(\mathrm{E})$;

(ii) A soft set I(E) of soft elements of $\mathfrak{A}$ (SBA) is called soft right ideal if it is no null soft set and has the following properties:

(a)If $\widetilde{\mathrm{x}}^{\mathrm{e}}, \tilde{\mathrm{y}}^{\mathrm{e}} \widetilde{\in} \mathrm{I}(\mathrm{E})$ then $\widetilde{\mathrm{x}}^{\mathrm{e}} \widetilde{\mp} \tilde{\mathrm{y}}^{\mathrm{e}} \widetilde{\in} \mathrm{I}(\mathrm{E})$;

(b) If $\widetilde{\mathrm{X}}^{\mathrm{e}} \widetilde{\in} \mathrm{I}(\mathrm{E})$ and $\widetilde{\alpha} \widetilde{\in} \mathbb{C}(\mathrm{E})$ then $\tilde{\mathrm{X}}^{\mathrm{e}} \tilde{\alpha} \widetilde{\alpha} \widetilde{\mathrm{I}}(\mathrm{E})$;

(c)If $\widetilde{\mathrm{X}}^{\mathrm{e}} \widetilde{\in} \mathrm{I}(\mathrm{E})$ and $\widetilde{\mathrm{r}}^{\mathrm{e}} \widetilde{\in} \mathfrak{A}$ then $\tilde{\mathrm{X}}^{\mathrm{e}} \sim \widetilde{\mathrm{r}}^{\mathrm{e}} \widetilde{\in} \mathrm{I}(\mathrm{E})$;

(iii) I(E) is called soft two sided ideal in $\mathfrak{A}$ if $\mathrm{I}(\mathrm{E})$ is both soft left ideal and soft right ideal .

Definition (3.11):

A soft ideal $\mathrm{I}(\mathrm{E})$ of $\mathfrak{U}$ (SBA) is said to be proper if $\mathrm{I}(\mathrm{E}) \neq \mathfrak{A}$.

\section{Definition(3.12):}

Let $\mathfrak{A}$ is a (S. B. A), a soft proper ideal $\mathcal{M}(\mathrm{E})$ of $\mathfrak{A}$ is said to be soft maximal left (or right, or two sided) ideal if there is no soft left (or right, or two sided) ideal $\mathcal{J}(\mathrm{E})$ in $\mathfrak{A}$ with $\mathcal{M}(\mathrm{E}) \underset{\subsetneq}{\widetilde{J}}(\mathrm{E}) \widetilde{\subsetneq} \mathfrak{A}$.

Definition (3.13):

The soft set of all soft maximal ideals $\mathcal{M}$ of given soft commutative banach algebra $\mathfrak{A}$ with $\tilde{\mathrm{u}}^{\mathrm{e}}$ by $\mathfrak{M}$. Every soft ideal $\mathcal{M} \widetilde{\in} \mathfrak{M}$ generates a soft homomorphism of $\mathfrak{A}$ onto $\mathbb{C}(E)$, we denote the soft number corresponding to the soft element $\widetilde{\mathrm{X}}^{\mathrm{e}} \widetilde{\in} \mathfrak{A}$ under this soft homomorphism by $\widetilde{\mathrm{x}}^{\mathrm{e}}(\mathcal{M})$. For fixed $\tilde{\mathrm{x}}^{\mathrm{e}} \widetilde{\in} \mathfrak{A}$ we obtain in this way soft function $\tilde{\mathrm{x}}^{\mathrm{e}}(\mathcal{M})$ on the soft set $\mathfrak{M}$. Consequently, we obtain a correspondence $\widetilde{\mathrm{x}}^{\mathrm{e}} \widetilde{\rightarrow} \widetilde{\mathrm{x}}^{\mathrm{e}}(\mathcal{M})$ between the soft element $\widetilde{\mathrm{x}}^{\mathrm{e}}$ of $\mathfrak{A}$ and soft functions $\widetilde{\mathrm{x}}^{\mathrm{e}}(\mathcal{M})$ on the soft set $\mathfrak{M}$.

I.e. the natural soft homomorphism

७: $\mathfrak{A} \rightarrow \frac{\mathfrak{A}}{\mathcal{M}}=\mathbb{C}(\mathrm{E})$ Defined by

$\vartheta\left(\widetilde{\mathrm{x}}^{\mathrm{e}}\right)=\widetilde{\mathrm{x}}^{\mathrm{e}} \widetilde{+} \mathcal{M}$ assign to each $\widetilde{\mathrm{x}}^{\mathrm{e}} \widetilde{\in} \mathfrak{A}$, a soft complex number $\tilde{\mathrm{x}}^{\mathrm{e}}(\mathcal{M})$ defined by

$\tilde{\mathrm{x}}^{\mathrm{e}}(\mathcal{M})=\tilde{\mathrm{x}}^{\mathrm{e}} \widetilde{+} \mathcal{M}$.

\section{Theorem (3.14):}

The correspondence $\widetilde{\mathrm{x}}^{\mathrm{e}} \widetilde{\rightarrow} \widetilde{\mathrm{x}}^{\mathrm{e}}(\mathcal{M})$ has the following properties:

(i) $\left(\tilde{x}^{e} \widetilde{+} \tilde{y}^{e}\right) \mathcal{M}=\tilde{\mathrm{x}}^{\mathrm{e}}(\mathcal{M}) \widetilde{+} \tilde{\mathrm{y}}^{\mathrm{e}}(\mathcal{M})$.

(ii)For $\widetilde{\alpha} \widetilde{\in} \mathbb{C}(E)$, then

$\left(\widetilde{\alpha} \tilde{\mathrm{x}}^{\mathrm{e}}\right)(\mathcal{M})=\widetilde{\alpha}\left(\tilde{\mathrm{x}}^{\mathrm{e}}(\mathcal{M})\right)$.

(iii) $\left(\tilde{\mathrm{x}}^{\mathrm{e}} \cdot \tilde{\mathrm{y}}^{\mathrm{e}}\right) \mathcal{M}=\tilde{\mathrm{x}}^{\mathrm{e}}(\mathcal{M}) \cdot \tilde{\mathrm{y}} \tilde{\mathrm{y}}^{\mathrm{e}}(\mathcal{M})$.

(iv) $\tilde{\mathrm{u}}^{\mathrm{e}}(\mathcal{M})=\overline{\widetilde{\mathrm{u}}^{\mathrm{e}}}$.

(v) $\tilde{\mathrm{x}}^{\mathrm{e}}(\mathcal{M})=\overline{\Theta^{\mathrm{e}}}$ if and only if $\tilde{\mathrm{x}}^{\mathrm{e}} \widetilde{\in} \mathcal{M}$.

(vi) $\left|\widetilde{\mathrm{x}}^{\mathrm{e}}(\mathcal{M})\right| \widetilde{\leq}\left\|\widetilde{\mathrm{x}}^{\mathrm{e}}\right\|$, for all $\widetilde{\mathrm{x}}^{\mathrm{e}} \widetilde{\in} \mathfrak{A}$.

Proof:

(i)

$\left(\tilde{\mathrm{x}}^{\mathrm{e}} \widetilde{+} \tilde{\mathrm{y}}^{\mathrm{e}}\right) \mathcal{M}=\tilde{\mathrm{x}}^{\mathrm{e}} \tilde{+} \tilde{\mathrm{y}}^{\mathrm{e}} \widetilde{+\mathcal{M}}=$

$\left(\tilde{\mathrm{x}}^{\mathrm{e}} \widetilde{\mathcal{M}}\right) \widetilde{f}\left(\tilde{\mathrm{y}}^{\mathrm{e}} \widetilde{\mathcal{H}}\right)=\tilde{\mathrm{x}}^{\mathrm{e}}(\mathcal{M}) \widetilde{\mp} \tilde{\mathrm{y}}^{\mathrm{e}}(\mathcal{M})$.

(ii) - for $\widetilde{\alpha} \widetilde{\in} \mathbb{C}(E),\left(\widetilde{\alpha} \tilde{\mathrm{x}}^{\mathrm{e}}\right)(\mathcal{M})=\widetilde{\alpha} \widetilde{\mathrm{x}}^{\mathrm{e}} \widetilde{+} \mathcal{M}$

$=\widetilde{\alpha}\left(\widetilde{\mathrm{x}}^{\mathrm{e}} \widetilde{+} \mathcal{M}\right)=\widetilde{\alpha}\left(\tilde{\mathrm{x}}^{\mathrm{e}}(\mathcal{M})\right)$. (iii) $\left(\tilde{\mathrm{x}}^{\mathrm{e}} \cdot \tilde{\mathrm{y}}^{\mathrm{e}}\right) \mathcal{M}=\left(\tilde{\mathrm{x}}^{\mathrm{e}} \cdot \tilde{\mathrm{y}}^{\mathrm{e}}\right) \widetilde{+} \mathcal{M}$

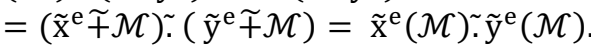

(iv) $\tilde{\mathrm{u}}^{\mathrm{e}}(\mathcal{M})=\tilde{\mathrm{u}}^{\mathrm{e}} \widetilde{+\mathcal{M}}={\overline{\mathrm{u}^{\mathrm{e}}}}$.

(v) $\tilde{\mathrm{x}}^{\mathrm{e}}(\mathcal{M})=\overline{\Theta^{\mathrm{e}}}$ iff $\tilde{\mathrm{x}}^{\mathrm{e}} \widetilde{+} \mathcal{M}=\mathcal{M}$ iff $\tilde{\mathrm{x}}^{\mathrm{e}} \widetilde{\in} \mathcal{M}$.

(vi) $\quad\left|\widetilde{\mathrm{x}}^{\mathrm{e}}(\mathcal{M})\right|=\left|\tilde{\mathrm{x}}^{\mathrm{e}} \widetilde{\mathcal{H}} \mathcal{M}\right|=\inf \left\{\left\|\tilde{\mathrm{x}}^{\mathrm{e}} \widetilde{\mathcal{M}}\right\|: \tilde{\mathrm{x}}^{\mathrm{e}}\right.$

$\widetilde{\in} \mathfrak{U}\} \widetilde{\leq}\left\|\widetilde{\mathrm{x}}^{\mathrm{e}}\right\|$, for all $\tilde{\mathrm{x}}^{\mathrm{e}} \widetilde{\in} \mathfrak{A}$.

\section{Definition (3.15):}

Let $\mathfrak{A}$ be Soft gelfand algebra and $\mathcal{M}$ be the soft set of all soft maximal ideals of $\mathfrak{U}$. Now for all $\tilde{\mathrm{x}}^{\mathrm{e}}$ $\widetilde{\in} \mathfrak{A}$, define $\widehat{\widetilde{\mathbf{x}}^{\mathrm{e}}}: \mathcal{M} \rightarrow \mathbb{C}(E)$ by $\widetilde{\widetilde{\mathrm{x}}}^{\mathrm{e}}(M)=\widetilde{\alpha}$ for all $M \widetilde{\in} \mathcal{M}$ where

$\widetilde{\mathrm{X}}^{\mathrm{e}}(M)=\widetilde{\mathrm{X}}^{\mathrm{e}}(M)=\widetilde{\mathrm{x}}^{\mathrm{e}} \widetilde{\mathrm{F}} \mathrm{M}=\widetilde{\alpha}$ also

$\mathbb{C}(E)=\frac{\mathfrak{A}}{\mathcal{M}}=\left\{\tilde{\mathrm{x}}^{\mathrm{e}} \widetilde{+} \mathcal{M}: \tilde{\mathrm{x}}^{\mathrm{e}} \widetilde{\in} \mathfrak{U}\right\}$

$=\{\widetilde{\alpha}: \widetilde{\alpha} \widetilde{\in} \mathbb{C}(E)\}$. Denoted $\widehat{\mathfrak{U}}=\left\{\widetilde{\mathrm{X}}^{\mathrm{e}}: \widetilde{\mathrm{x}}^{\mathrm{e}} \widetilde{\in} \mathfrak{U}\right\}$.

Theorem (3.16):

Let $\mathfrak{A}$ be (S. B. A) with $\tilde{\mathrm{u}}^{\mathrm{e}}$ and let $\mathbb{f}_{1}, \mathbb{f}_{2}$ be two soft multiplicative functions on $\mathfrak{U}$ and $\operatorname{ker} \mathbb{f}_{1}=\operatorname{ker} \mathbb{f}_{2}$ then $\mathbb{f}_{1}=\mathbb{f}_{2}$.

Proof:

Suppose that $\operatorname{kerf} \mathbb{f}_{1}=\operatorname{kerf}_{2}=\mathcal{M}$.

Since $\mathbb{f}_{1}, \mathbb{f}_{2}$ are soft multiplicative

$\Rightarrow \mathbb{f}_{1} \neq \Theta^{\mathrm{e}}, \mathbb{f}_{2} \neq \Theta^{\mathrm{e}} \Rightarrow \exists \widetilde{\mathrm{X}}^{\mathrm{e}}{ }_{0} \widetilde{\in} \mathfrak{A}$ Such that $\mathbb{f}_{1}\left(\widetilde{\mathrm{X}}_{0}{ }_{0}\right) \neq \Theta^{\mathrm{e}}$

$\Rightarrow \widetilde{\mathrm{x}}_{0}^{\mathrm{e}} \widetilde{\notin} \operatorname{kerf} \mathbb{f}_{1}=\mathcal{M}$. Now for all $\tilde{\mathrm{x}}^{\mathrm{e}} \widetilde{\in} \mathfrak{A}$.

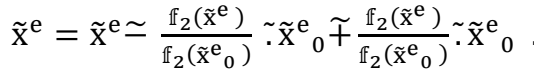

Now, $\quad \mathbb{F}_{2}\left(\tilde{\mathrm{x}}^{\mathrm{e}} \simeq \frac{\mathbb{f}_{2}\left(\tilde{\mathrm{x}}^{\mathrm{e}}\right)}{\mathbb{f}_{2}\left(\tilde{\mathrm{x}}^{\mathrm{e}}{ }_{0}\right)} \sim \tilde{\mathrm{x}}_{0}^{\mathrm{e}}\right) \mathbb{f}_{2}\left(\tilde{\mathrm{x}}^{\mathrm{e}}\right)-\frac{\mathbb{f}_{2}\left(\tilde{\mathrm{x}}^{\mathrm{e}}\right)}{\mathbb{f}_{2}\left(\tilde{\mathrm{x}}^{\mathrm{e}}{ }_{0}\right)}$

$\tilde{.} \mathbb{f}_{2}\left(\tilde{\mathrm{X}}_{0}^{\mathrm{e}}\right)=\Theta^{\mathrm{e}}$

$\Rightarrow \widetilde{\mathrm{x}}^{\mathrm{e}} \simeq \frac{\mathbb{f}_{2}\left(\widetilde{\mathrm{x}}^{\mathrm{e}}\right)}{\mathbb{f}_{2}\left(\widetilde{\mathrm{x}}^{\mathrm{e}}{ }_{0}\right)} \sim \tilde{\mathrm{x}}_{0}^{\mathrm{e}} \widetilde{\in} \operatorname{ker} \mathbb{f}_{2}=\mathcal{M}$

$\Rightarrow \tilde{\mathrm{x}}^{\mathrm{e}} \simeq \frac{\mathbb{f}_{2}\left(\tilde{\mathrm{x}}^{\mathrm{e}}\right)}{\mathbb{1}_{2}\left(\tilde{\mathrm{x}}^{\mathrm{e}}{ }_{0}\right)} \sim \widetilde{\mathrm{x}}_{0}^{\mathrm{e}}=m$, for some

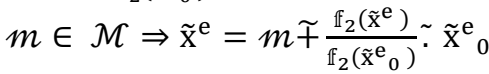

$\Rightarrow \widetilde{\mathrm{x}}^{\mathrm{e}}=m \widetilde{+} \beta \sim \widetilde{\mathrm{x}}_{0}^{\mathrm{e}}$ where $\beta=\frac{\mathbb{f}_{2}\left(\widetilde{\mathrm{x}}^{\mathrm{e}}\right)}{\mathbb{f}_{2}\left(\tilde{\mathrm{x}}^{\mathrm{e}}{ }_{0}\right)} \Rightarrow \mathbb{F}_{1}\left(\widetilde{\mathrm{x}}^{\mathrm{e}}\right)=$

$\mathbb{f}_{1}(m) \widetilde{+} \beta \mathbb{f}_{1}\left(\widetilde{\mathrm{x}}_{0}^{\mathrm{e}}\right)$

$=\Theta \mathrm{e} \widetilde{+} \frac{\mathbb{f}_{2}\left(\tilde{\mathrm{x}}^{\mathrm{e}}\right)}{\mathbb{f}_{2}\left(\tilde{\mathrm{x}}^{\mathrm{e}}{ }_{0}\right)} \sim \mathbb{f}_{1}\left(\tilde{\mathrm{x}}_{0}^{\mathrm{e}}\right)$

$\Rightarrow \mathbb{f}_{1}\left(\tilde{\mathrm{x}}^{\mathrm{e}}\right)=\alpha \tilde{\alpha} \mathbb{f}_{2}\left(\tilde{\mathrm{x}}^{\mathrm{e}}\right) \quad$ where $\alpha=\frac{\mathbb{f}_{1}\left(\tilde{\mathrm{x}}_{0} \mathrm{e}\right)}{\mathbb{f}_{2}\left(\tilde{\mathrm{x}}_{0}\right)}$.

Two show that $\alpha=\tilde{\mathrm{u}}^{\mathrm{e}}$, consider

$$
\begin{aligned}
& \alpha \tilde{\alpha}\left(\mathbb{F}_{2}\left(\tilde{\mathrm{x}}^{\mathrm{e}}\right)\right)^{2}=\alpha \tilde{\alpha} \mathbb{f}_{2}\left(\tilde{\mathrm{x}}^{\mathrm{e}}\right) \tilde{\mathbb{F}_{2}}\left(\tilde{\mathrm{x}}^{\mathrm{e}}\right) \\
& =\alpha \cdot \tilde{\mathbb{f}_{2}}\left(\tilde{\mathrm{x}}^{\mathrm{e}^{2}}\right)=\mathbb{f}_{1}\left(\tilde{\mathrm{x}}^{\mathrm{e}}\right) \\
& =\left(\alpha \cdot \mathbb{f}_{2}\left(\tilde{\mathrm{x}}^{\mathrm{e}}\right)\right)^{2}=\alpha^{2 \sim} \cdot\left(\mathbb{f}_{2}\left(\tilde{\mathrm{x}}^{\mathrm{e}}\right)\right)^{2}
\end{aligned}
$$

$\Rightarrow \tilde{\alpha .}\left(\mathbb{F}_{2}\left(\tilde{\mathrm{x}}^{\mathrm{e}}\right)\right)^{2}=\alpha^{2 \sim} .\left(\mathbb{F}_{2}\left(\tilde{\mathrm{x}}^{\mathrm{e}}\right)\right)^{2}$

$\Rightarrow\left(\alpha^{2} \simeq \alpha\right) \sim\left(\mathbb{f}_{2}\left(\tilde{\mathrm{x}}^{\mathrm{e}}\right)\right)^{2}=\Theta^{\mathrm{e}}$

$\Rightarrow \alpha^{2} \simeq \alpha=\Theta^{\mathrm{e}}$ as $\mathbb{f}_{2}\left(\tilde{\mathrm{x}}^{\mathrm{e}}\right) \neq \Theta^{\mathrm{e}} \Rightarrow \alpha=\Theta^{\mathrm{e}}$ or

$\alpha=\tilde{\mathrm{u}}^{\mathrm{e}}$, if $\alpha=\Theta^{\mathrm{e}}$

$\Rightarrow \mathbb{f}_{1}\left(\tilde{\mathrm{x}}^{\mathrm{e}}\right)=\Theta^{\mathrm{e}}$

This is contradiction, then $\alpha=\tilde{\mathrm{u}}^{\mathrm{e}}$.

Theorem (3.17):

There is a soft bijective corresponding between the soft set of all soft maximal ideals of $\mathfrak{A}$ and the soft set of all soft characters of $\mathfrak{A}$. 
Noori .F / Hayder .K

\section{Proof:}

Let $\mathcal{M}$ be soft set of all maximal ideals of $\mathfrak{A}$ and $\mathcal{C}$ be soft set of all soft characters of $\mathfrak{A}$. Define $\mathfrak{Q}: \mathcal{M} \rightarrow \mathcal{C}$ by $\mathfrak{Q}(\mathcal{M})=\mathbb{f}_{\mathcal{M}}$ such that $\mathbb{f}_{\mathcal{M}}\left(\tilde{\mathrm{x}}^{\mathrm{e}}\right)=$ $\tilde{\mathrm{x}}^{\mathrm{e}}(\mathcal{M})=\tilde{\mathrm{x}}^{\mathrm{e}} \tilde{+} \mathcal{M}$. Let $\mathcal{M}_{1}=\mathcal{M}_{2}$, $\mathcal{M}_{1}$ and $\mathcal{M}_{2} \widetilde{\in} \mathcal{M}$

$\Rightarrow \widetilde{\mathrm{x}}^{\mathrm{e}} \widetilde{\mathcal{H}} \mathcal{M}_{1}=\widetilde{\mathrm{x}}^{\mathrm{e}} \widetilde{\mathrm{T}} \mathcal{M}_{2} \Rightarrow \widetilde{\mathrm{x}}^{\mathrm{e}}\left(\mathcal{M}_{1}\right)=\widetilde{\mathrm{x}}^{\mathrm{e}}\left(\mathcal{M}_{2}\right) \Rightarrow$ $\mathbb{f}_{\mathcal{M}_{1}}=\mathbb{f}_{\mathcal{M}_{2}}$

$\Rightarrow \mathfrak{Q}\left(\mathcal{M}_{1}\right)=\mathfrak{Q}\left(\mathcal{M}_{2}\right) \Rightarrow \mathfrak{Q}$ is well define

Let $\quad \mathfrak{Q}\left(\mathcal{M}_{1}\right)=\mathfrak{Q}\left(\mathcal{M}_{2}\right) \Rightarrow \mathbb{f}_{\mathcal{M}_{1}}=\mathbb{f}_{\mathcal{M}_{2}} \Rightarrow$ $\tilde{\mathrm{x}}^{\mathrm{e}}\left(\mathcal{M}_{1}\right)=\widetilde{\mathrm{x}}^{\mathrm{e}}\left(\mathcal{M}_{2}\right)$

$\Rightarrow \widetilde{\mathrm{x}}^{\mathrm{e}} \widetilde{+} \mathcal{M}_{1}=\widetilde{\mathrm{x}}^{\mathrm{e}} \widetilde{+} \mathcal{M}_{2} \Rightarrow \mathcal{M}_{1}=\mathcal{M}_{2} \Rightarrow \mathfrak{Q}$ is soft injective.

To show that $\mathfrak{Q}$ is soft Surjective, for $\mathbb{f} \widetilde{\in} \mathcal{C}$, to show $\mathbb{f}=\mathbb{f}_{\mathcal{M}_{0}}$ for some $\mathcal{M}_{0} \widetilde{\in} \mathcal{M}$. Let

$\mathcal{M}_{0}=\operatorname{ker} \mathbb{f}=\left\{\widetilde{\mathrm{x}}^{\mathrm{e}} \widetilde{\in} \mathfrak{U}: \mathbb{f}\left(\widetilde{\mathrm{x}}^{\mathrm{e}}\right)=\Theta^{\mathrm{e}}\right\}$, now $\mathcal{M}_{0}$ is soft proper ideal of $\mathfrak{U}$. If not $\Rightarrow \tilde{\mathrm{u}}^{\mathrm{e}} \widetilde{\in} \mathcal{M}_{0} \Rightarrow$ $\mathbb{f}\left(\tilde{\mathrm{u}}^{\mathrm{e}}\right)=\Theta^{\mathrm{e}}$ this contradiction

To show that $\mathcal{M}_{0}$ is soft maximal.

Let $\mathcal{M}_{0} \widetilde{\subsetneq} \mathcal{J}$ to show that $\mathcal{J}=\mathfrak{A}$, now $\mathcal{J} \subseteq \mathfrak{A}$ and let $\widetilde{\mathrm{x}}^{\mathrm{e}} \widetilde{\in} \mathfrak{A}$, since $\mathcal{M}_{0} \widetilde{\subsetneq} \mathcal{J}$

$\Rightarrow \exists \widetilde{\mathrm{x}}_{0}^{\mathrm{e}} \widetilde{\in} \mathcal{J}$ and $\widetilde{\mathrm{x}}_{0}{ }_{0} \widetilde{\notin} \mathcal{M}_{0}$, now $\widetilde{\mathrm{x}}^{\mathrm{e}}$

$=\widetilde{\mathrm{x}}^{\mathrm{e}} \simeq \frac{\mathbb{f}\left(\tilde{\mathrm{x}}^{\mathrm{e}}\right)}{\mathbb{f}\left(\tilde{\mathrm{x}}^{\mathrm{e}}{ }_{0}\right)} \sim \widetilde{\mathrm{x}}^{\mathrm{e}}{ }_{0} \widetilde{+} \frac{\mathbb{f}\left(\tilde{\mathrm{x}}^{\mathrm{e}}\right)}{\mathbb{f}\left(\tilde{\mathrm{x}}^{\mathrm{e}}{ }_{0}\right)} \sim \widetilde{\mathrm{x}}^{\mathrm{e}}{ }_{0}$

$\Rightarrow \mathbb{f}\left(\widetilde{\mathrm{X}}^{\mathrm{e}} \simeq \frac{\mathbb{f}\left(\tilde{\mathrm{x}}^{\mathrm{e}}\right)}{\mathbb{f}\left(\tilde{\mathrm{x}}_{0}\right)} \sim \widetilde{\mathrm{x}}_{0}^{\mathrm{e}}\right)$

$=\mathbb{f}\left(\widetilde{\mathrm{X}}^{\mathrm{e}}\right) \simeq \frac{\mathbb{f}\left(\tilde{\mathrm{x}}^{\mathrm{e}}\right)}{\mathbb{f}^{\mathrm{f}}\left(\tilde{\mathrm{x}}_{0}\right)} \sim \mathbb{f}\left(\widetilde{\mathrm{X}}_{0}^{\mathrm{e}}\right)=\Theta^{\mathrm{e}}$

$\Rightarrow \widetilde{\mathrm{x}} \mathrm{e} \simeq \frac{\mathbb{f}\left(\tilde{\mathrm{x}}^{\mathrm{e}}\right)}{\mathbb{f}\left(\tilde{\mathrm{x}}^{\mathrm{e}}{ }_{0}\right)} \sim \widetilde{\mathrm{x}}_{0}^{\mathrm{e}}{ }_{0} \widetilde{\in} \operatorname{ker} \mathbb{f}=\mathcal{M}_{0}$

$\Rightarrow \widetilde{\mathrm{x}}^{\mathrm{e}} \simeq \frac{\mathbb{f}\left(\tilde{\mathrm{x}}^{\mathrm{e}}\right)}{\mathbb{f}\left(\tilde{\mathrm{x}}^{\mathrm{e}}\right)} \sim \widetilde{\mathrm{x}}_{0}^{\mathrm{e}}=\mathrm{m}$, for some $\mathrm{m} \widetilde{\in} \mathcal{M}_{0}$

$\Rightarrow \tilde{\mathrm{x}}^{\mathrm{e}}=\mathrm{m} \widetilde{+} \alpha \cdot \tilde{\mathrm{X}}^{\mathrm{e}}{ }_{0}$, where $\alpha \widetilde{\in} \mathbb{C}(\mathrm{E})$ and

$\alpha=\frac{\mathbb{f}\left(\widetilde{\mathrm{x}}^{\mathrm{e}}\right)}{\mathbb{f}\left(\tilde{\mathrm{x}}^{\mathrm{e}}{ }_{0}\right)}$. Now $\widetilde{\mathrm{x}}^{\mathrm{e}}{ }_{0} \widetilde{\in} \mathcal{J}$ and $\alpha \widetilde{\in} \mathbb{C} €$

$\Rightarrow \tilde{\alpha} \cdot \widetilde{\mathrm{x}}^{\mathrm{e}}{ }_{0} \widetilde{\in} \mathcal{J}$ also $\mathrm{m} \widetilde{\in} \mathcal{M}_{0} \Rightarrow \widetilde{\mathrm{x}}^{\mathrm{e}} \widetilde{\in} \mathcal{J}$

$\Rightarrow \mathcal{J}=\mathfrak{A} \Rightarrow \mathcal{M}_{0}$ is soft maximal.

To show that ker $\mathbb{f}=\operatorname{ker} \mathbb{f} \mathcal{M}_{0}$

$\operatorname{ker} \mathbb{f}_{\mathcal{M}_{0}}=\left\{\widetilde{\mathrm{X}}^{\mathrm{e}} \widetilde{\in} \mathfrak{U}: \mathbb{f}_{\mathcal{M}_{0}}\left(\widetilde{\mathrm{X}}^{\mathrm{e}}\right)=\overline{\Theta^{\mathrm{e}}}\right\}$

$\left\{\tilde{\mathrm{X}}^{\mathrm{e}} \widetilde{\in} \mathfrak{U}: \tilde{\mathrm{x}}^{\mathrm{e}}\left(\mathcal{M}_{0}\right)=\overline{\Theta^{\mathrm{e}}}\right\}$

$=\left\{\tilde{\mathrm{x}}^{\mathrm{e}} \widetilde{\in} \mathfrak{A}: \tilde{\mathrm{x}}^{\mathrm{e}} \widetilde{\mathcal{H}} \mathcal{M}_{0}=\mathcal{M}_{0}\right\}$

$=\left\{\widetilde{\mathrm{X}}^{\mathrm{e}} \widetilde{\in} \mathfrak{A}: \widetilde{\mathrm{X}}^{\mathrm{e}} \widetilde{\in} \mathcal{M}_{0}\right\}=\operatorname{ker} \mathbb{f}$

$\Rightarrow \mathbb{f}=\mathbb{f}_{\mathcal{M}_{0}}$ (by theorem3) $\Rightarrow \mathfrak{Q}$ is soft surjective and hence $\mathfrak{Q}$ is a soft bijective.

\section{Theorem (3.18):}

Let $\mathfrak{A}$ be Soft gelfand algebra then for all $\widetilde{\mathrm{x}}^{\mathrm{e}} \widetilde{\in} \mathfrak{A}$

$$
\begin{aligned}
& \text { (i) }\left\|\tilde{\mathrm{x}}^{\mathrm{e}^{2}}\right\|=\left\|\tilde{\mathrm{x}}^{\mathrm{e}}\right\|^{2} . \\
& \text { (ii) } \mathrm{r}\left(\widetilde{\mathrm{x}}^{\mathrm{e}}\right)=\left\|\tilde{\mathrm{x}}^{\mathrm{e}}\right\| . \\
& \text { (iii) }\left\|\widetilde{\mathrm{x}}^{\mathrm{e}}\right\|=\left\|\tilde{\mathrm{x}}^{\mathrm{e}}\right\| .
\end{aligned}
$$

Proof:

(i) $\Rightarrow(\boldsymbol{i} i)$

Suppose that $\left\|\tilde{\mathrm{x}}^{\mathrm{e}}\right\|=\left\|\widetilde{\mathrm{x}}^{\mathrm{e}}\right\|^{2}$, for all $\tilde{\mathrm{X}}^{\mathrm{e}} \widetilde{\in} \mathfrak{A}$, then in general we have

$\left\|\tilde{\mathrm{x}}^{\mathrm{e}^{2 \mathrm{k}}}\right\|=\left\|\tilde{\mathrm{x}}^{\mathrm{e}}\right\|^{2 \mathrm{k}}, \mathrm{k}=0,1,2, \ldots$.
Since $r\left(\widetilde{\mathrm{x}}^{\mathrm{e}}\right)=\lim _{\mathrm{n} \rightarrow \infty}\left\|\tilde{\mathrm{x}}^{\mathrm{e}}\right\|^{\frac{1}{\mathrm{n}}}$ (soft spectral radius formula), and $\left\{\left\|\tilde{\mathrm{x}}^{\mathrm{e}^{2 \mathrm{k}}}\right\|=\left\|\tilde{\mathrm{x}}^{\mathrm{e}}\right\|^{2 \mathrm{k}}\right\}$ is sub sequence of the sequence $\left\{\left\|\tilde{x}^{e^{n}}\right\|^{\frac{1}{n}}\right\}$, we have

$$
r\left(\tilde{\mathrm{x}}^{\mathrm{e}}\right)=\lim _{\mathrm{k} \rightarrow \infty}\left\|\tilde{\mathrm{x}}^{\mathrm{e}^{2 \mathrm{k}}}\right\|^{\frac{1}{2 \mathrm{k}}} \quad=\lim _{\mathrm{k} \rightarrow \infty}\left(\left\|\tilde{\mathrm{x}}^{\mathrm{e}}\right\|^{2 \mathrm{k}}\right)^{\frac{1}{2 \mathrm{k}}}=
$$

$\left\|\tilde{\mathrm{x}}^{\mathrm{e}}\right\|$.

(ii) $\Rightarrow(\boldsymbol{i})$

Suppose that $\mathrm{r}\left(\tilde{\mathrm{x}}^{\mathrm{e}}\right)=\left\|\tilde{\mathrm{x}}^{\mathrm{e}}\right\|$

$\Rightarrow \mathrm{r}\left(\tilde{\mathrm{x}}^{\mathrm{e}^{2}}\right)=\left\|\tilde{\mathrm{x}}^{\mathrm{e}}\right\|$, and by theorem (3.2)

$r\left(\tilde{\mathrm{x}}^{\mathrm{e}^{2}}\right)=\left(\mathrm{r}\left(\tilde{\mathrm{x}}^{\mathrm{e}}\right)\right)^{2} \Rightarrow\left(\mathrm{r}\left(\tilde{\mathrm{x}}^{\mathrm{e}}\right)\right)^{2}=\left\|\tilde{\mathrm{x}}^{\mathrm{e}}\right\| \quad$ and also $r\left(\tilde{\mathrm{x}}^{\mathrm{e}}\right)=\left\|\tilde{\mathrm{x}}^{\mathrm{e}}\right\| \Rightarrow\left(\mathrm{r}\left(\tilde{\mathrm{x}}^{\mathrm{e}}\right)\right)^{2}=\left\|\tilde{\mathrm{x}}^{\mathrm{e}}\right\|^{2}$.

(ii) $\Leftrightarrow(\boldsymbol{i i i})$

Suppose that $r\left(\tilde{\mathrm{x}}^{\mathrm{e}}\right)=\left\|\tilde{\mathrm{x}}^{\mathrm{e}}\right\|$, iff

$\mathrm{r}\left(\widetilde{\mathrm{X}}^{\mathrm{e}}\right)=\operatorname{Sup}\left\{|\widetilde{\alpha}|: \widetilde{\alpha} \widetilde{\in} \delta\left(\widetilde{\mathrm{x}}^{\mathrm{e}}\right)\right\}$

$=\operatorname{Sup}\left\{\left|\widetilde{\mathrm{x}}^{\mathrm{e}}(\mathrm{M})\right|: \mathrm{M} \widetilde{\in} \mathcal{M}\right\}=\left\|\widetilde{\mathrm{x}}^{\mathrm{e}}\right\|$.

\section{References}

[1] Molodtsov, D.,"Soft set theory - first results", Computers Mathematics with Appl., 37(1999), 1931.

[2] Maji K., Biswas R. and Roy R., "Soft set theory", Computers Mathematics with Appl., 45(2003), 555-562.

[3] Cagman N. and Enginoglu S., "Soft set theory and uni-int decision making", European J. Oper. Res., 207(2010), 848-855.

[4]. Muhammad S. and Munazza N., "On soft topological spaces ", Computers Mathematics with Appl., 61(2011), 1786-1799.

[5] Sujoy D. and Smanta D.,"Soft metric", Ann. fuzzy math. Inf., pp. 1-18, 2013.

[6] Sujoy D. and Smanta S., "Soft real sets, soft real numbers and their properties", J. fuzzy math. , 20(2012), 551-576.

[7] Das S. and Smanta S., "on Soft complex sets, soft complex numbers ", J. fuzzy math., 21(2013), 195-216.

[8] Sujoy D., Majumadar, P. and Smanta S.,"on soft linear spaces and soft normed linear spaces", Ann. Fuzzy math. Inform.9 (2015), 91-109.

[9] Thakur R. and Smanta S.," Soft Banach Algebra", Ann. Fuzzy Math. And inf., 10 (2015), 397-412.

[10] Petroudi S., Sadati S. and Yaghobi A.,

"New Results on Soft Banach Algebra", Int., J. Sci. and Engineering Investigations, 6(2017) 17-25.

[11] Shbeeb, M., H.," Some Results on Soft Topological Spaces ", Ph.D. Dissertation, AlMustansiriya University, 2015.

[12] Maji P. K., Biswas R. and Roy A. R., "Soft set theory", Computers and Mathematics with Applications, 45(2003), 555-562. 


$$
\text { نتائج جديدة حول الطيف الواهن في بناخ الجبرا الواهنة }
$$

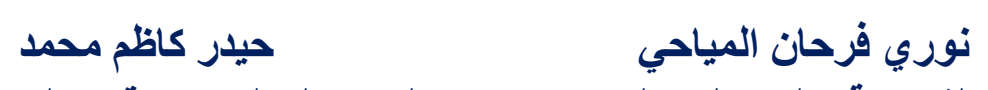

جامعه القادية/ كليه علوم الحاسوب وتكنولوجيا المعلومات/ قسم الرياضيات

Header.Kadim@gmail.com $\quad$ Nfam60@yahoo.com

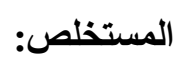

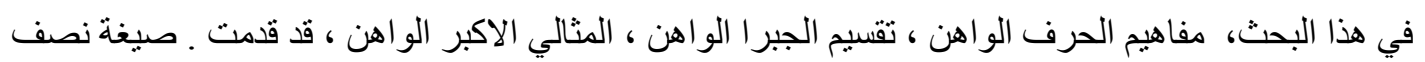

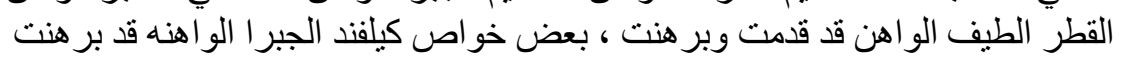

